\title{
No Independent Association of Circulating Fetuin-A with Insulin Sensitivity in Young Women
}

\section{(요 $(3 \ominus$}

Authors

Sabrina Reif1, 2, 3, Sarah Moschko', 2, 3, Christina Gar ${ }^{1,2,3}$, Uta Ferrari1, 2, 3, 4, Nina Hesse ${ }^{5}$, Nora N. Sommer ${ }^{5}$, Jochen Seißler'1, 2, 3, Andreas Lechner ${ }^{1,2,3}$

Affiliations

1 Diabetes Research Group, Medizinische Klinik und Poliklinik IV, Klinikum der Universität München, München, Germany

2 Clinical Cooperation Group Type 2 Diabetes, Helmholtz Zentrum München, Neuherberg, Germany

3 German Center for Diabetes Research (DZD), Neuherberg, Germany

4 Department of Geriatrics, Medizinische Klinik und Poliklinik IV, Klinikum der Universität München, München, Germany

5 Klinik und Poliklinik für Radiologie, Klinikum der Universität München, München, Germany

Key words type 2 diabetes, metabolic syndrome, non-alcoholic fatty liver disease, mineral metabolism

received 20.01.2020

accepted after revision $\quad$ 08.07.2020

published online 11.05 .2020

\section{Bibliography}

Horm Metab Res 2020; 52: 809-814

DOI 10.1055/a-1216-4405

ISSN 0018-5043

(c) 2020. The Author(s)

This is an open access article published by Thieme under the terms of the Creative Commons Attribution-NonDerivative-NonCommercial-License, permitting copying and reproduction so long as the original work is given appropriate credit. Contents may not be used for commercial purpose, or adapted, remixed, transformed or built upon. (https://creativecommons. org/licenses/by-nc-nd/4.0/)

Georg Thieme Verlag KG, Rüdigerstraße 14,

70469 Stuttgart, Germany
Correspondence

Andreas Lechner MD

Medizinische Klinik und Poliklinik IV, Klinikum der Universität München

Ziemssenstraße 1

80336 München

Germany

Tel.: +49894400 52288, Fax: +4989440054956

丹 Supplementary material is available under https://doi.org/10.1055/a-1216-4405.

\section{ABSTRACT}

Animal data link high circulating fetuin-A to low insulin sensitivity and observational studies identify the hepatokine as a marker of future incident type 2 diabetes mellitus in humans. However, a recent, well-powered Mendelian randomization study finds no causal role. We therefore tested in a deeply-phenotyped human cohort if circulating fetuin-A correlates independently with insulin sensitivity and how it relates to the metabolic syndrome and ectopic fat deposition. We analyzed data from 290 young women with and without recent gestational diabetes mellitus. We found that circulating fetuin-A correlates inversely with insulin sensitivity in univariate analyses, but that this correlation is lost after adjustment for markers of the metabolic syndrome and of fatty liver. Additionally, we investigated which fat compartment associates most strongly with circulating fetuin-A. In whole body MRI data from a subcohort of 152 women, this was liver fat content. We conclude that high circulating fetuin-A occurs as part of the metabolic syndrome in young women and associates most strongly with liver fat content. Its close link to the metabolic syndrome may also cause the inverse correlation of circulating fetuin-A with insulin sensitivity as we found no independent association.

\section{Introduction}

Fetuin-A (alpha-Heremans-Schmidt-Glycoprotein; AHSG) is a protein, which is abundantly secreted into the bloodstream by the liver and is mainly involved in mineral trafficking [1].
Additional functions seen in vitro and in animal studies, are a suppression of adiponectin production [2], the induction of subclinical inflammation [3], and a non-competitive inhibition of the insulin receptor tyrosine kinase [4]. In some of these functions, fetuin-A was found to interact with non-esterified fatty acids (NEFA) 
[5]. Combined, these functions can reduce insulin sensitivity and thus potentially link fetuin-A to the pathogenesis of type 2 diabetes mellitus (T2DM).

However, whether high levels of fetuin-A cause insulin resistance and T2DM in humans is still unclear. In several cross-sectional studies, its circulating concentration correlated inversely with insulin sensitivity [6-8], but this finding was not universal $[8,9]$ and the correlation was always weakened when adjustments for conventional metabolic risk factors were included. Fetuin-A was also found to be higher in prediabetic subjects compared to normoglycemic controls [10] and we previously showed the same for women after gestational diabetes mellitus (GDM) compared to women after a normoglycemic pregnancy [11]. Furthermore, high circulating fetuin-A appeared to be an independent risk factor for future T2DM in several prospective, observational studies [12]. High fetuin-A and low adiponectin were found to be a particularly predictive combination [13]. On the other hand, a recent, well-powered Mendelian randomization study could not confirm a causal relationship [14].

The uncertainty about whether or not fetuin-A interferes directly with human glucose metabolism is caused by the fact that high circulating concentrations of the protein associate with fatty liver and other components of the metabolic syndrome [6]. Because this syndrome, as a whole, lowers insulin sensitivity and increases the risk for T2DM, the assessment of the specific role of fetuin-A is problematic. Full adjustment for other metabolic syndrome-related risk factors would be a prerequisite for this kind of analysis, but this is often impossible in large cohort studies with limited phenotypic information. These difficulties can lead to an overestimation of fetuin-A's direct effect $[12,14]$.

To overcome this problem, we examined the association of circulating fetuin-A with insulin sensitivity in a deeply-phenotyped human cohort, where the potentially confounding factors were recorded in detail. Using this approach, we found no independent negative correlation of fetuin-A with insulin sensitivity.

\section{Subjects and Methods}

\section{Study cohort}

We analyzed data from the baseline visit of the prospective, monocenter, observational cohort study PPS Diab ('Prediction, Prevention and Subclassification of Type 2 Diabetes Study'). Participants were enrolled between November 2011 and May 2016. The study population consists of women with GDM during their last pregnancy (post GDM group) and women following a normoglycemic pregnancy (control group) in the ratio 2:1. The cohort was recruited consecutively from the diabetes center and the obstetrics department of the University Hospital (Klinikum der Universität München) in Munich, Germany.

Eligible women were premenopausal and within 3 to 16 months after a singleton or twin $(n=9)$ pregnancy with live birth(s). The diagnosis of GDM was based on a $75 \mathrm{~g}$ oral glucose tolerance test (OGTT). The cut-off values for GDM were $92 / 180 / 153 \mathrm{mg} / \mathrm{dl}$ plasma glucose following the International Association of the Diabetes and Pregnancy Study Group (IADPSG) recommendations. Women could participate as controls if they had no history of GDM in any previous pregnancy and either a normal $75 \mathrm{~g}$ OGTT or a normal $50 \mathrm{~g}$ screening OGTT (<135 mg/dl plasma glucose after $1 \mathrm{~h}, \mathrm{n}=10$ ) after the $23^{\text {rd }}$ week of gestation.

Exclusion criteria for this study were alcohol or substance abuse, pre-pregnancy diabetes and chronic diseases requiring continuous medication, except for hypothyroidism $(n=52)$, bronchial asthma $(n=8)$, mild hypertension $(n=4)$, gastroesophageal reflux $(n=2)$, and history of pulmonary embolism resulting in Rivaroxaban prophylaxis $(n=1)$.

Written informed consent was obtained from all study participants and the protocol was approved by the ethical review committee of the Ludwig-Maximilians-Universität (study ID 300-11).

\section{Main outcomes}

We focused on the correlation of the fasting fetuin-A level in plasma with insulin sensitivity, determined during an OGTT immediately following the fasting blood draw. We examined this correlation with and without adjustment for other components of the metabolic syndrome.

\section{Measurements}

History, questionnaires, anthropometrics, blood sampling, and OGTT

All study participants provided a detailed medical history. Weight, height, waist, and hip circumference were determined using standard protocols [11]. Blood pressure was measured in a seated position. Mean blood pressure (bp) was calculated as (systolic pressure + diastolic pressure $\times 2$ )/3. A fasting blood draw and 5-point $75 \mathrm{~g}$ OGTT were done in the morning after at least $10 \mathrm{~h}$ of fasting.

Plasma glucose, triglycerides, HDL cholesterol and gamma glutamyl transferase were determined in a routine clinical chemistry laboratory. Insulin was measured in serum by a chemoluminescence immunoassay (DiaSorin Liaison; Saluggia, Italy), fetuin-A in EDTA plasma by an ELISA (BioVendor, Heidelberg, Germany). The insulin sensitivity index (ISI) was calculated according to Matsuda and de Fronzo [15]. The rise in serum insulin from 0 to $30 \mathrm{~min}$ of the OGTT ( $\triangle$ INS $30^{\prime}$ ) was used as the measure of insulin secretion. This approach was previously validated using intravenous GTT data [11].

MRI

All study participants were invited to undergo a whole-body MRI scan (3 Tesla system, Ingenia, or Achieva; Philips Healthcare, Best, Netherlands). A detailed MRI protocol was published previously [11].

In brief, liver fat content was determined by fat-fraction maps generated from a modified two-point Dixon sequence. Three regions of interest with sizes of approximately $120-150 \mathrm{~mm}^{2}$ each were placed in segment VII in the right liver lobe. Visceral adipose tissue volume (VAT) was determined on a three-dimensional mDixon or an axial $\mathrm{T} 1$ weighted whole body sequence using the segmentation software SliceOmatic 4.3 rev-11 (TomoVision, Magog, Canada).

\section{Statistical analysis}

Metric variables are presented as median (1. quartile; 3. quartile), categorial variable as number (percentage). The Spearman correlation coefficient $(\rho)$ is used for univariate correlations. For multi- 
- Table 1 Baseline characteristics of the study cohort $(n=290)$.

\begin{tabular}{|c|c|c|}
\hline Age (years) & \multicolumn{2}{|c|}{$35(33 ; 38)$} \\
\hline Time since delivery (months) & \multicolumn{2}{|c|}{$9.1(7.3 ; 11.8)$} \\
\hline \multirow[t]{5}{*}{ Glucose tolerance at study visit } & NGT & $210(72.4 \%)$ \\
\hline & IFG & $35(12.1 \%)$ \\
\hline & IGT & $26(9.0 \%)$ \\
\hline & IFG + IGT & $12(4.1 \%)$ \\
\hline & T2DM & $7(2.4)$ \\
\hline \multirow{2}{*}{$\begin{array}{l}\text { Glucose tolerance during } \\
\text { Preceding pregnancy }\end{array}$} & GDM & $188(64.8 \%)$ \\
\hline & NGT & $102(35.2 \%)$ \\
\hline BMI $\left(\mathrm{kg} / \mathrm{m}^{2}\right)$ & \multicolumn{2}{|c|}{$23.6(21.4 ; 27.1)$} \\
\hline$W C(\mathrm{~cm})$ & \multicolumn{2}{|c|}{$79(73 ; 87)$} \\
\hline Mean BP (mmHg) & \multicolumn{2}{|c|}{$87(82 ; 94)$} \\
\hline$|S|$ & \multicolumn{2}{|c|}{$5.3(3.4 ; 7.5)$} \\
\hline$\Delta \mathrm{INS} 30^{\prime}(\mathrm{mU} / \mathrm{l})$ & \multicolumn{2}{|c|}{$44.0(31.6 ; 67.5)$} \\
\hline Triglycerides (mg/dl) & \multicolumn{2}{|c|}{$68(54 ; 92)$} \\
\hline HDL cholesterol (mg/dl) & \multicolumn{2}{|c|}{$62(53 ; 72)$} \\
\hline NEFA $(\mu \mathrm{mol} / \mathrm{l})$ & \multicolumn{2}{|c|}{$601(479 ; 746)$} \\
\hline GGT (U/I) & \multicolumn{2}{|c|}{$14(11 ; 19)$} \\
\hline Fetuin-A ( $\mu \mathrm{g} / \mathrm{ml})$ & \multicolumn{2}{|c|}{$238(214 ; 263)$} \\
\hline Adiponectin $(\mu \mathrm{g} / \mathrm{ml})$ & \multicolumn{2}{|c|}{$11.27(7.81 ; 14.89)$} \\
\hline Liver fat content ( $\% ; n=152$ ) & \multicolumn{2}{|c|}{$0.43(0 ; 1.36)$} \\
\hline $\operatorname{VAT}(I ; n=152)$ & \multicolumn{2}{|c|}{$1.63(1.01 ; 2.64)$} \\
\hline
\end{tabular}

BMI: Body mass index; WC: Waist circumference; Mean BP: Mean blood pressure; ISI: Insulin sensitivity index; $\triangle \mathrm{INS} 30^{\prime}$ : Rise in serum insulin from 0 to $30 \mathrm{~min}$ of the OGTT; NEFA: Non-esterified fatty acids; GGT: Gamma glutamyl transferase; VAT: Abdominal visceral adipose tissue volume.

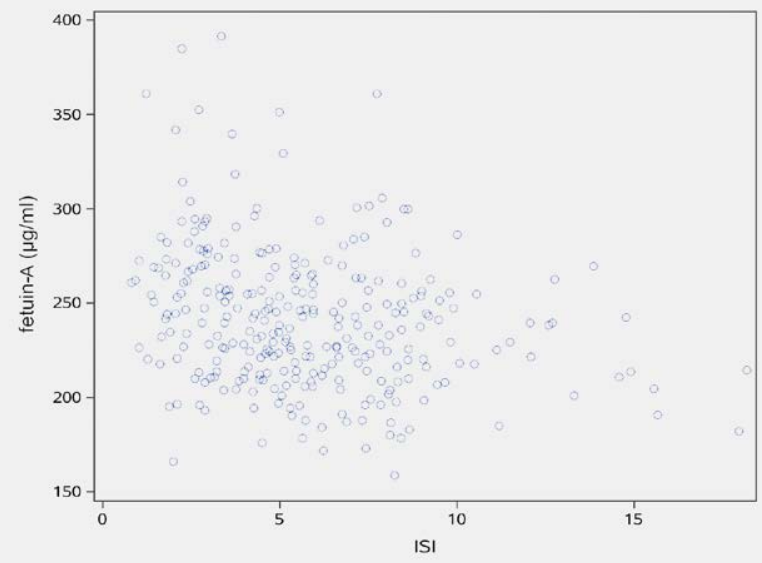

- Fig. 1 Scatterplot of insulin sensitivity index (ISI) and circulating fetuin-A; $\rho=-0.26 p=<0.0001$.

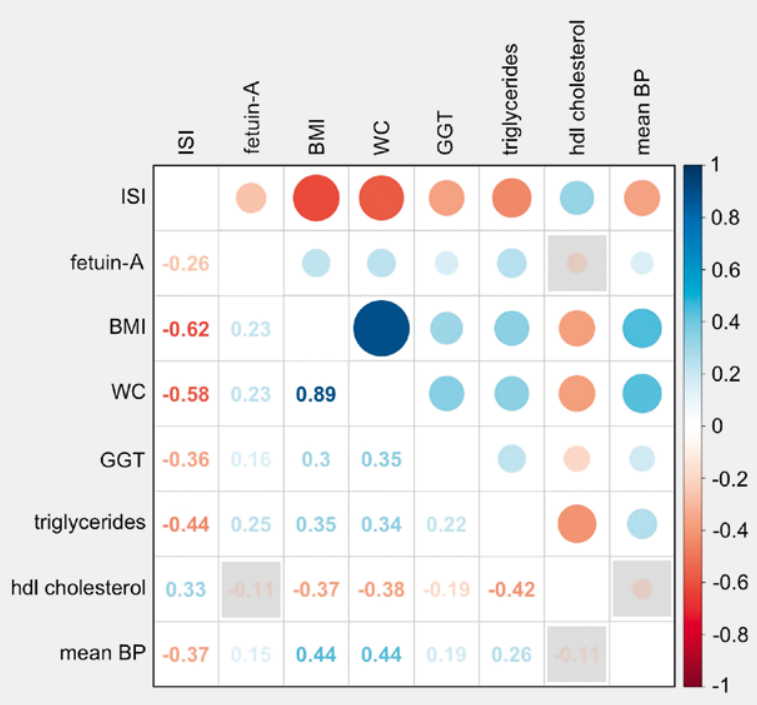

- Fig. 2 Correlation matrix of main study variables; visual representation of the correlations in the upper right part, spearman correlation coefficients in the lower left part; shaded squares mark correlations with $p$-values $\geq 0.05$, all others have a $p$-value $<0.05$; $\mathrm{n}=290$. ISI: Insulin sensitivity index; BMI: Body mass index; WC: Waist circumference; GGT: Gamma glutamyl transferase; Mean BP: Mean blood pressure

- Table 2 Different linear regression models with the dependent variable $\log |S|$.

\begin{tabular}{|l|c|c|c|}
\hline & $\begin{array}{l}\text { Adjusted } \\
\mathbf{R}^{2}\end{array}$ & $\begin{array}{l}\text { Standardized } \\
\text { beta coefficient }\end{array}$ & p-Value \\
\hline Fetuin-A & 0.07 & -0.27 & $<0.0001$ \\
\hline Fetuin-A & 0.45 & -0.14 & 0.002 \\
\hline BMI & & -0.63 & $<0.0001$ \\
\hline Fetuin-A & 0.43 & -0.14 & 0.002 \\
\hline WC & & -0.61 & $<0.0001$ \\
\hline Fetuin-A & 0.46 & -0.12 & 0.006 \\
\hline WC & & -0.55 & $<0.0001$ \\
\hline GGT & & -0.20 & $<0.0001$ \\
\hline Fetuin-A & 0.51 & -0.08 & 0.08 \\
\hline WC & & -0.47 & $<0.0001$ \\
\hline GGT & & -0.17 & 0.0001 \\
\hline Triglycerides & & -0.25 & $<0.0001$ \\
\hline Fetuin-A & 0.51 & -0.07 & 0.1 \\
\hline WC & & -0.44 & $<0.0001$ \\
\hline GGT & & -0.17 & $<0.0001$ \\
\hline Triglycerides & & -0.24 & $<0.0001$ \\
\hline HDL cholesterol & & 0.02 & 0.6 \\
\hline Mean BP & & -0.05 & 0.3 \\
\hline ISI: Insulin sensitivity index; BMI: Body mass index; WC: Waist \\
\hline blood pressure. & & & \\
\hline & & & \\
\hline & & & \\
\hline
\end{tabular}


- Table 3 Different linear regression models with the dependent variable log ISI.

\begin{tabular}{|c|c|c|c|c|c|c|}
\hline \multirow[b]{2}{*}{ Subgroup } & \multicolumn{2}{|c|}{ Adjusted $\mathbf{R}^{2}$} & \multicolumn{2}{|c|}{ Standardized beta coefficient } & \multicolumn{2}{|l|}{ p-Value } \\
\hline & $<$ & $\geq$ & $<$ & $\geq$ & $<$ & $\geq$ \\
\hline Fetuin-A & 0.03 & 0.12 & -0.19 & -0.36 & 0.02 & $<0.0001$ \\
\hline Fetuin-A & 0.35 & 0.54 & -0.10 & -0.18 & 0.14 & 0.003 \\
\hline BMI & & & -0.58 & -0.67 & $<0.0001$ & $<0.0001$ \\
\hline Fetuin-A & 0.34 & 0.52 & -0.10 & -0.18 & 0.14 & 0.003 \\
\hline WC & & & -0.56 & -0.66 & $<0.0001$ & $<0.0001$ \\
\hline Fetuin-A & 0.37 & 0.54 & -0.08 & -0.16 & 0.19 & 0.007 \\
\hline WC & & & -0.51 & -0.60 & $<0.0001$ & $<0.0001$ \\
\hline GGT & & & -0.22 & -0.15 & 0.001 & 0.02 \\
\hline Fetuin-A & 0.44 & 0.57 & -0.06 & -0.10 & 0.32 & 0.09 \\
\hline WC & & & -0.46 & -0.50 & $<0.0001$ & $<0.0001$ \\
\hline GGT & & & -0.16 & -0.15 & 0.02 & 0.01 \\
\hline Triglycerides & & & -0.27 & -0.22 & 0.0001 & 0.001 \\
\hline Fetuin-A & 0.43 & 0.57 & -0.08 & -0.10 & 0.25 & 0.11 \\
\hline WC & & & -0.46 & -0.45 & $<0.0001$ & $<0.0001$ \\
\hline GGT & & & -0.17 & -0.16 & 0.01 & 0.008 \\
\hline Triglycerides & & & -0.25 & -0.21 & 0.0008 & 0.003 \\
\hline HDL cholesterol & & & 0.06 & 0.01 & 0.42 & 0.85 \\
\hline Mean BP & & & 0.03 & -0.1 & 0.64 & 0.14 \\
\hline
\end{tabular}

Subgroups of study participants with NEFA levels < and $\geq$ of the cohort median. ISI: Insulin sensitivity index; BMI: Body mass index; WC: Waist circumference; GGT: Gamma glutamyl transferase; Mean BP: Mean blood pressure.

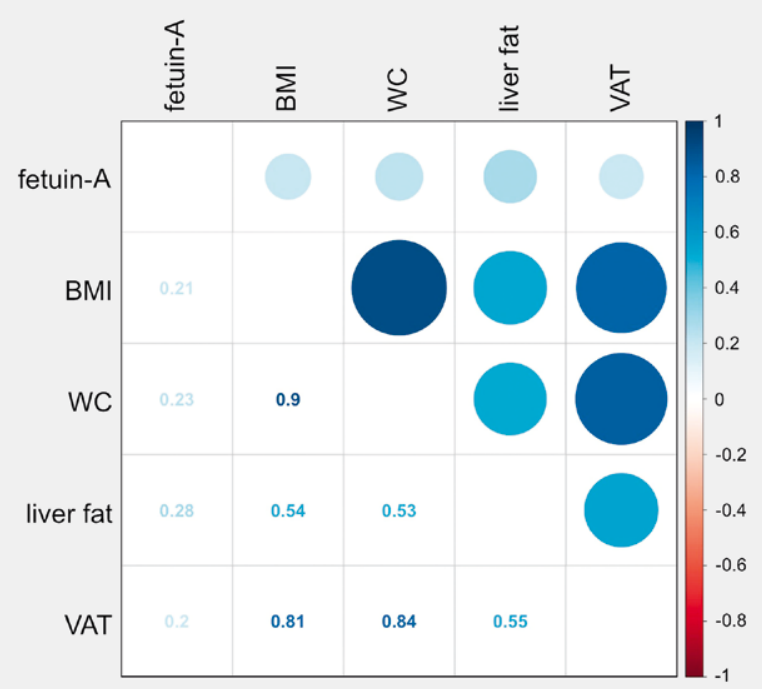

- Fig. 3 Correlation matrix of fetuin-A, conventional measures of adiposity [BMI: waist circumference (WC)], MRI-measured liver fat content and abdominal visceral adipose tissue volume (VAT); visual representation of the correlations in the upper right part, Spearman correlation coefficients in the lower left part; all p-values $<0.05$; $\mathrm{n}=152$. WC: Waist circumference. variate linear regressions, log ISI was used as the dependent variable. All analyses were performed with SAS version 9.4.

\section{Results}

A total of 304 women from the PPS Diab study cohort completed the baseline visit. Out of these, we excluded two because of type 1 diabetes diagnosed during follow-up, two because of overt hyperthyroidism, one because of an acute upper respiratory infection at baseline and nine because of at least one missing value for BMI, waist circumference, triglycerides, HDL cholesterol, mean bp or ISI. The final sample therefore consisted of 290 women. They were 35 $(33 ; 38)$ years old, had a BMI of $23.6 \mathrm{~kg} / \mathrm{m}^{2}(21.4 ; 27.1)$ and an ISI of $5.3(3.4 ; 7.5)$. During the preceding pregnancy, $188(64.8 \%)$ of the women had had GDM and at the time of the study visit, 73 (24.2\%) had prediabetes and 7 (2.4\%) had newly-diagnosed T2DM. The full baseline characteristics are shown in $>$ Table 1.

In univariate correlation analyses, fetuin-A correlated negatively with ISI ( $\rho=-0.26 ; p=<0.0001$; $>$ Fig. 1$)$ and positively with BMI $(\rho=0.23 ; p=0.0001)$, waist circumference (WC; $p=0.23 ; p<0.0001)$, gamma glutamyl transferase (GGT; $\rho=0.16 ; p=0.007$ ), triglycerides $(\rho=0.25 ; p<0.0001)$ and mean bp $(p=0.15 ; p=0.01)$ ( Fig. 2$)$.

In a univariate linear regression analysis, fetuin-A associated with $\log$ ISI $(\beta=-0.27 ; p<0.0001)$. This association was weakened with adjustment for BMI, WC or the combination of WC and GGT, 
as a surrogate marker for fatty liver, but remained significant. However, with adjustment for WC, GGT and triglycerides, as well as in the full model with WC, GGT, triglycerides, HDL cholesterol and mean bp, fetuin-A was no longer a significantly associated with log ISI ( $\triangleright$ Table 2). Fetuin-A did not associate with early insulin secretion in the OGTT $\left(\triangle \mathrm{INS} 30^{\prime} ; \beta=0.11 ; \mathrm{p}=0.054\right)$.

Because fetuin-A was previously shown to interact with high NEFA [5] and low adiponectin [13] concentrations in the plasma, we repeated the regression analyses of $>$ Table 2 in the subgroups of study participants with below and at or above median levels of NEFA or adiponectin, respectively. While the results were similar for high and low adiponectin ( $\triangleright$ Table 1S), the associations of fetuin-A with log ISI were stronger for high than for low NEFA ( $\triangleright$ Table 3). However, as in the whole study cohort, fetuin-A no longer associated significantly with log ISI with adjustment for WC, GGT, and triglycerides, as well as in the full model with WC, GGT, triglycerides, HDL cholesterol, and mean bp.

We also measured liver fat content and body fat distribution by MRI in a subcohort of 152 women ( $\mathbf{F i g}$. 3). Here, fetuin-A correlated most strongly with liver fat content $(\rho=0.28 ; p=0.0005)$ and less strongly with waist circumference ( $\rho=0.23 ; p=0.005)$, BMI $(\rho=0.21 ; p=0.01)$ and abdominal visceral adipose tissue volume $(\rho=0.2 ; p=0.02)$.

\section{Discussion}

The main findings of this cross-sectional analysis in young women were that circulating fetuin-A correlates inversely with insulin sensitivity, as shown previously, but that this is not an independent correlation. Rather, elevated fetuin-A appears to be just one component of the metabolic syndrome, which, as a whole, causes insulin resistance.

Our findings disagree with previous publications that found an independent, negative association of fetuin-A with insulin sensitivity $[5-9,13]$. One possible reason for this disagreement is the different structure of our cohort compared to the previous studies. With only premenopausal women and a relatively low median liver fat content in our cohort, the association of fetuin-A with insulin sensitivity may have been weakened. We observed similar results for FGF-21 in another hepatokine we measured in this cohort (data not shown). An alternative reason for the disagreement of this study with previous publications may be that different variables were included in the final multivariate models. We could confirm that high fetuin-A associates with insulin resistance more closely in the presence of high plasma NEFA [5]. This effect did not make fetuin-A an independent factor after multivariate adjustment but it was nevertheless noticeable ( $>$ Table $\mathbf{3}$ ).

In addition to the results discussed so far, our analysis of MRI data confirmed the specific link of high circulating fetuin-A and fatty liver, which had already been shown previously [6]. This link may tie fatty liver to bone mineralization and vascular calcification because fetuin- $A$ is involved in both processes [1]. This connection warrants further investigation.

The strengths of our study include its cohort that is homogeneous for sex and age but has a wide range of BMI and metabolic disease risk. Additionally, phenotyping in this prospective cohort was precise and in depth and preanalytic procedures were optimized to obtain high quality biosamples. The weaknesses of our study include its cross-sectional, observational design that precludes the definitive determination of cause-effect relationships. Additionally, our focus on young women, although a strength with respect to a clean analysis, does not permit generalization to other segments of the population.

We conclude that high circulating fetuin-A is linked to metabolic syndrome and hepatic steatosis in young women and is also associated with low insulin sensitivity. This association, however, is not independent and therefore the importance of fetuin- $A$ in this segment of the population remains unclear. Further investigations of the relevant cause-effect relationships are warranted. Additionally, plasma fetuin-A may be a helpful biomarker for metabolic syndrome in young women with a particular relevance in context of gestational diabetes mellitus.

\section{Funding Information}

German Center for Diabetes Research

Helmholtz Zentrum München

Klinikum der Universität München

\section{Conflict of Interest}

The authors declare that they have no conflict of interest.

\section{References}

[1] Holt SG, Smith ER. Fetuin-A-containing calciprotein particles in mineral trafficking and vascular disease. Nephrol Dial Transplant 2016; 31: 1583-1587

[2] Hennige AM, Staiger $\mathrm{H}$, Wicke $\mathrm{C}$ et al. Fetuin-A induces cytokine expression and suppresses adiponectin production. PloS one 2008; 3: e1765

[3] Pal D, Dasgupta S, Kundu R et al. Fetuin-A acts as an endogenous ligand of TLR4 to promote lipid-induced insulin resistance. Nat Med 2012; 18: 1279-1285

[4] Srinivas PR, Deutsch DD, Mathews ST et al. Recombinant human alpha 2-HS glycoprotein inhibits insulin-stimulated mitogenic pathway without affecting metabolic signalling in Chinese hamster ovary cells overexpressing the human insulin receptor. Cell Signal 1996; 8: 567-573

[5] Stefan N, Haring HU. Circulating fetuin-A and free fatty acids interact to predict insulin resistance in humans. Nat Med 2013; 19: 394-395

[6] Stefan N, Hennige AM, Staiger $\mathrm{H}$ et al. Alpha2-Heremans-Schmid glycoprotein/fetuin-A is associated with insulin resistance and fat accumulation in the liver in humans. Diabetes Care 2006; 29: 853-857

[7] Erdmann J, Salmhofer H, Knauss A et al. Relationship of fetuin-A levels to weight-dependent insulin resistance and type 2 diabetes mellitus. Regul Peptides 2012; 178: 6-10

[8] Ishibashi A, Ikeda Y, Ohguro T et al. Serum fetuin-A is an independent marker of insulin resistance in Japanese men. J Atheroscler Thromb 2010; 17: 925-933

[9] Mori K, Emoto M, Yokoyama $\mathrm{H}$ et al. Association of serum fetuin-A with insulin resistance in type 2 diabetic and nondiabetic subjects. Diabetes Care 2006; 29: 468 
[10] Ou HY, Yang YC, Wu HT et al. Increased fetuin-A concentrations in impaired glucose tolerance with or without nonalcoholic fatty liver disease, but not impaired fasting glucose. J Clin Endocrinol Metab 2012; 97: 4717-4723

[11] Rottenkolber M, Ferrari U, Holland L et al. The diabetes risk phenotype of young women with recent gestational diabetes. J Clin Endocrinol Metab 2015; 100: E910-E918

[12] Sujana C, Huth C, Zierer A et al. Association of fetuin-A with incident type 2 diabetes: results from the MONICA/KORA Augsburg study and a systematic meta-Analysis. Eur J Endocrinol 2018; 178: 389-398
[13] Stefan N, Sun Q, Fritsche A et al. Impact of the adipokine adiponectin and the hepatokine fetuin-A on the development of type 2 diabetes: Prospective cohort- and cross-sectional phenotyping studies. PLoS One 2014; 9: e92238

[14] Kroger ], Meidtner K, Stefan N et al. Circulating fetuin-A and risk of type 2 diabetes: A mendelian randomization analysis. Diabetes 2018; 67: 1200-1205

[15] Matsuda M, DeFronzo RA. Insulin sensitivity indices obtained from oral glucose tolerance testing: Comparison with the euglycemic insulin clamp. Diabetes Care 1999; 22: 1462-1470 


\section{Supplementary Material}

- Table $1 S$ Different linear regression models with the dependent variable log ISI; Subgroups of study participants with adiponectin levels < and $\geq$ the cohort median.

\begin{tabular}{|c|c|c|c|c|c|c|}
\hline \multirow{2}{*}{ subgroup } & \multicolumn{2}{|c|}{ Adjusted $R^{2}$} & \multicolumn{2}{|c|}{ Standardized beta coefficient } & \multicolumn{2}{|l|}{ p-value } \\
\hline & $<$ & $\geq$ & $<$ & $\geq$ & $<$ & $\geq$ \\
\hline fetuin-A & 0.06 & 0.05 & -0.26 & -0.24 & 0.002 & 0.004 \\
\hline fetuin-A & 0.51 & 0.29 & -0.15 & -0.12 & 0.01 & 0.10 \\
\hline BMI & & & -0.68 & -0.50 & $<0.0001$ & $<0.0001$ \\
\hline fetuin-A & 0.50 & 0.22 & -0.15 & -0.14 & 0.02 & 0.06 \\
\hline WC & & & -0.67 & -0.43 & $<0.0001$ & $<0.0001$ \\
\hline fetuin-A & 0.54 & 0.24 & -0.13 & -0.12 & 0.02 & 0.10 \\
\hline WC & & & -0.60 & -0.41 & $<0.0001$ & $<0.0001$ \\
\hline GGT & & & -0.21 & -0.15 & 0.0006 & 0.05 \\
\hline fetuin- $A$ & 0.58 & 0.32 & -0.07 & -0.11 & 0.22 & 0.12 \\
\hline WC & & & -0.54 & -0.29 & $<0.0001$ & 0.0002 \\
\hline GGT & & & -0.21 & -0.07 & 0.0005 & 0.37 \\
\hline triglycerides & & & -0.24 & -0.33 & 0.0001 & $<0.0001$ \\
\hline fetuin- $A$ & 0.58 & 0.32 & -0.07 & -0.09 & 0.21 & 0.19 \\
\hline WC & & & -0.55 & -0.27 & $<0.0001$ & 0.001 \\
\hline GGT & & & 0.20 & -0.08 & 0.0006 & 0.32 \\
\hline triglycerides & & & 0.23 & -0.32 & 0.0004 & 0.0002 \\
\hline hdl cholesterol & & & 0.04 & -0.02 & 0.53 & 0.82 \\
\hline mean $\mathrm{BP}$ & & & 0.04 & -0.1 & 0.60 & 0.20 \\
\hline
\end{tabular}

$\mathrm{ISI}=$ insulin sensitivity index; $\mathrm{BMI}=$ body mass index; $\mathrm{WC}=$ waist circumference; $\mathrm{GGT}=$ gamma glutamyl transferase; mean $\mathrm{BP}=$ mean blood pressure . 